\title{
El efecto mediador de la capacidad ejecutiva para la innovación entre la gestión del conocimiento y el rendimiento de la cadena de suministros
}

\author{
Rafael E. Apolinario ${ }^{1,2}$ y Daniel E. Guevara ${ }^{1}$ \\ (1) CENTRUM Catolica Graduate Business School - Pontificia Universidad Catolica del Peru, Lima, Peru. \\ (correo-e: a20152120@pucp.pe; dguevaras@pucp.pe) \\ (2) Facultad de Ciencias Administrativas, Universidad de Guayaquil, Ciudadela Universitaria, \\ Av. Detla y Av. Kennedy, Ecuador. (correo-e: rafael.apolinarioqu@ug.edu.ec)
}

Recibido Ago. 7, 2020; Aceptado Oct. 7, 2020; Versión final Nov. 19, 2020, Publicado Feb. 2021

\begin{abstract}
Resumen
El objetivo del presente estudio fue determinar el efecto mediador de la capacidad ejecutiva para la innovación, entre la gestión del conocimiento y el rendimiento de la cadena de suministros, en empresas pymes (pequeñas y medianas empresas) del sector logístico portuario. Se analizaron los patrones de gestión en empresas pymes con más de cinco años, cuyas actividades estén directamente relacionadas con el manejo de la cadena logística del Ecuador. Este estudio se encuentra estructurado en un marco literario donde se abordan las variables: gestión del conocimiento, capacidad ejecutiva para la innovación y el rendimiento de la cadena de suministros. La técnica de validación del modelo propuesto son ecuaciones estructurales mediante estimaciones basadas en la función de máxima verosimilitud. Se encontró que existe una mediación parcial entre las variables. Se concluye que las pymes necesitan capacidad innovativa para mejorar su gestión del conocimiento y su rendimiento en la cadena de suministros.
\end{abstract}

Palabras clave: gestión del conocimiento; pequeñas y medianas empresas; sector logístico portuario; cadena de suministros

\section{Mediating effect of executive capacity for innovation between knowledge management and supply chain performance}

\begin{abstract}
The objective of the present study was to determine the mediating effect of executive capacity for innovation between knowledge management and supply chain performance in SMEs (small and medium-sized enterprises) from the port logistics sector. Management pattern variables were assessed in SMEs that were at least five years in business and that had performed activities directly related to managing Ecuador's logistics chain. A literature review was the framework for the present study where the following variables were examined: knowledge management, executive capacity for innovation, and supply chain performance. Applying structural equations through estimates based on maximum likelihood validated the proposed model. The results showed that there was partial mediation between the examined variables. In conclusion, SMEs need innovative capacity to improve their supply chain's knowledge management and performance.
\end{abstract}

Keywords: knowledge management; small and medium-sized enterprises; port logistics sector; supply chain 


\section{INTRODUCCIÓN}

El Sector Logístico Portuario (SLP) representa un eslabón importante en la economía de los países que poseen sistemas portuarios (Cooper et al., 2016). Representando alrededor del 85\% de la carga mundial anual transportada y estando en constante crecimiento, proporciona una importante fuente de empleos de manera directa e indirecta, gracias a su dinamismo y relevancia en la diversas actividades económicas, destacándose sobre todo, las pequeñas y medianas empresas (pymes) quienes juegan un papel preponderante con sus operaciones y están en constante competitividad para mantenerse en este mercado (Antai, 2011), realizando grandes esfuerzos en el desempeño de sus competencias y habilidades en la cadena de suministros (CS), logrados a partir de los lazos comerciales que establecen en interacción continua con los agentes del sistema portuario (Davis et al., 2007). Por lo tanto, las pymes se ven en la necesidad de tener dentro de sus capacidades la capacidad de innovar, gestionar sus recursos propios y desarrollarse en el sector al que pertenecen, optimizando sus gastos y recursos para la reducción de costos, en espera de mantener su vida institucional en el mediano o largo plazo (Busse y Wallenburg, 2011).

Las pymes del SLP tienen varios desafíos para mantenerse en el mercado, debido a que están sometidas a la presión de que, por diferentes situaciones, pueda desembocar en su desaparición, resultado de las dificultades y desafíos sociales, económicos y de gestión, que normalmente se hallan en procesos de formación y evolución para su manutención (Buestán y Van-Landeghem, 2015). Llevados principalmente por la pobre evolución, entendimiento y fortalecimiento en lo que respecta a factores como la gestión del conocimiento (GC), la capacidad ejecutiva para la innovación (CEI) (Esper et al., 2010) y el rendimiento de la cadena de suministros (RCS), entre otras (Carrillo, 2007). Las pymes en general representan el $50 \%$ del PIB mundial, convirtiéndose en uno de los principales motores de desarrollo económico de los países. La importancia de realizar estudios que conlleven al desarrollo y aplicación de la GC, CEl y el RCS trascendiendo de lo teórico hasta llegar a lo social (Algorta y Zeballos, 2011), permitirán identificar en las pymes esquemas propios de revisión del RCS cuando estas maduren las características innatas de innovación que poseen.

A diferencia de las operadoras internacionales que, tienen la facilidad de adaptarse a los cambios y sus esfuerzos en nuevos contextos son mínimos en comparación con las pymes, estas últimas no pueden en su gran mayoría afrontar cambios bruscos por reformas de procesos, siendo particular el tipo de operación en que se especializan, al ofrecer servicios específicos como transportación, carga y descarga de mercaderías, servicios de operadoras de trámites, verificadoras, almaceneras, servicios prestados, entre otros; tienden a estancarse y enfocarse en un solo producto, pese a la flexibilización en los procesos y operaciones que el SLP proporciona a las industrias de todos los tamaños, permitiéndoles la búsqueda de facilidades competitivas (Galvao et al., 2016), que a su vez permitan abordar la relación entre GC y RCS a través de la mediación que proporciona la CEI en la teoría de capacidades dinámicas.

Wynarczyk (2013) y Vieera (2014) concuerdan en que los resultados de las evaluaciones en las pymes del sector de servicios tienen mayor dependencia en sus decisiones claves, lo que motiva su competitividad en el desarrollo de sus capacidades dinámicas organizacionales. Es decir, la habilidad de vincular las gestiones ejecutivas con el progreso y compromiso de las organizaciones influyen en el rigor de las decisiones sobre la flexibilidad e innovación de los procesos, al no asumir los riesgos en los períodos de crisis (Simangunsong et al., 2016). Por otro lado, el conocimiento y la innovación son esenciales para las organizaciones, más aún para las pymes debido a que constituyen la base de su sostenibilidad y supervivencia, permitiéndoles un rendimiento eficaz y eficiente (Eyaa et al., 2010; Cela et al., 2015; Chen y Kirsis, 2017). Por lo tanto, es importante identificar las principales fuentes de incertidumbre de las variables de estudio que permitan llevar a cabo modelos dirigidos a estas organizaciones (Kwak et al., 2018).

Estudios como los de Kearney et al. (2018), argumentan que la GC se relaciona con la capacitación y el aprendizaje teniendo en cuenta el mejoramiento continuo, la revitalización de las estrategias y las capacidades organizacionales para su desarrollo. De esta manera, autores como (Yousif y Hassan, 2013; Wynarczyk, 2013; Al- Hakim y Hassan, 2013; An et al., 2014; Liao y Barnes, 2015; Dahash, 2017; Ding et al., 2017; Echeverri et al., 2018) coinciden en considerar a la GC como un factor importante para la toma de decisiones en la implementación de las innovaciones a través de sus capacidades de ejecución. Por su parte, Agostini et al. (2017), destaca que la CEI y la RCS, son variables elementales en los procesos operacionales de la CS de cualquier industria. En consecuencia, el desempeño y el buen manejo de la CS en todas sus aplicaciones de su gestión, esto es, en los procesos de aprovisionamiento, fabricación y distribución, son las claves para una efectiva ventaja competitiva a través, de estrategias naturales en coordinación con sus clientes, proveedores y productores (Li et al., 2005).

Para este estudio se realizó una extensa revisión literaria, en la cual se indagaron tanto los procesos y capacidades dinámicas de las organizaciones, así como aquellas características coyunturales que presentan las pymes del SLP en la gestión y revisión de su cadena de suministros, durante el desarrollo innovativo de 
la firma. De lo cual se observan pocos estudios relacionados a las pymes en el SLP, evidenciando brechas entre la GC, CEI y RCS en circunstancia de que los mayores estudios están dirigidos a evaluar las relaciones con los consumidores y la dinámica propia de las grandes empresas industriales y comerciales de bienes (Esmer et al., 2016; Fantazy y Salem, 2016).

Por lo cual se analizó la GC bajo la visión de la teoría de la gestión del conocimiento basada en los recursos, misma que vincula a las estrategias, los recursos, la cultura y la infraestructura (Adegbuyi y Uhomoibhi, 2008; Vasconcelos, 2008; Algorta y Zeballos, 2011; Darroch, 2005; Cheng y Kuan, 2015); el RCS es valorado en términos de calidad, costo y puntualidad, considerándose para el efecto, la eficiencia y la eficacia, las que serán analizadas bajo una perspectiva de la cadena de suministro integral (Kearney et al., 2018). Y finalmente abordado el efecto mediador que presenta la CEI basada en la teoría de capacidades dinámicas, para las variables anteriores, tomando como punto de estudio las capacidades organizacionales, la revitalización de las estrategias y el mejoramiento continuo; que posibilitan la promoción o retención de las actividades de emprendimiento con el objeto de preservar el capital y la adaptación competitiva, optimizando de manera eficiente los procesos empresariales en todos sus niveles (Åslund y Bäckström, 2017; El Baz et al., 2019). De esta manera, se busca identificar la brecha entre estas variables, entre las gestiones administrativas y operacionales de las pymes que deben ser canalizadas desde sus diferentes departamentos para su existencia en los mercados dinámicos (Fletcher y Polychronakis, 2007; López y Meroño, 2011; Massingham, 2014; Kaliani et al., 2016; Galvao et al., 2016).

Este estudio se encuentra estructurado de un marco literario donde se abordan las variables: GC, CEI y RCS y además el contexto al que corresponde es el SLP, posterior se presenta las hipótesis de investigación con sus respectivos autores que las justifican, le sigue la metodología, la discusión de los resultados y en la parte final se presentan las conclusiones y las respectivas referencias bibliográficas.

\section{OTROS ANTECEDENTES}

Esta sección describe los marcos literarios de las variables de estudio junto con los constructos que se proponen, además se detallan las hipótesis con su justificación respectiva a cada una de ellas.

\section{Sector logístico portuario (SLP)}

La cadena de suministro es un proceso integrado, donde la materia prima, después de un proceso de transformación, se presenta en productos terminados para luego entregarlas a los clientes a través de la distribución, mediante cuatro eslabones que comprenden varios sistemas de intereses grandes y complejos, estos son (a) los suministros, (b) la fabricación, (c) la distribución, y (d) los consumidores (Chen y Kirsis, 2017). Eyaa et al. (2010) indican que los obstáculos que afectan el rendimiento en las pymes incluyen los inadecuados y costosos recursos que se traducen en las limitaciones de la inversión financiera y de los accesos a las redes de competitividad sustentable. Es así como Fantazy y Salem (2016) concluyen que los efectos positivos de la gestión de control de los RCS están en las estrategias. Así pues, de las investigaciones analizadas sobre estas relaciones se puede mencionar el estudio de Cheng y Kuan (2015) sobre el desarrollo, validación, y la medición del rendimiento en la GC, quienes proponen un modelo de medición para las pymes y luego, Kearney et al. (2018) desarrollan un marco teórico de la CEI en un contexto portuario.

Las pymes, con respecto a las grandes empresas, necesitan marcar la diferencia especialmente en los procesos de investigación e innovación operacional. Bajo este panorama, las pymes llegan a presentar controles rígidos en su estructura organizacional debido al significado e importancia que les otorgan a sus pequeñas inversiones. El nivel de producción influye directamente en los resultados de sus actividades, afectando su competitividad, la cual es determinada por la capacidad para innovar y desarrollarse en el sector al que pertenecen (Martínez et al., 2016; Hamid, 2018; Lee et al., 2019). Laperche y Liu (2013) indican que la dependencia de mercados vecinos, las recesiones económicas y la incapacidad de cambio en las pymes, influyen en la rápida desaceleración de la economía y la desaparición de las organizaciones que se desarrollan dentro del SLP. Por tal, se hace énfasis en los procesos de formación y desarrollo de las pymes que se fundamentan en información generada en etapas iniciales, en los cuales se puede evidenciar los grandes desafíos sociales y económicos que enfrentan, frenando, en muchos casos, su eficiencia y eficacia para mantener su existencia.

Zortea et al. (2012) evaluaron a las pymes que trabajan en el sector de servicios y observaron que estas muestran una mayor dependencia de la gerencia en sus decisiones claves tales como la motivación a la competitividad, el desarrollo de las capacidades dinámicas de investigación e innovación, la capacidad de gestión, y la habilidad de vincular a los gobiernos en el desarrollo y compromiso de las organizaciones. Por su lado, Wynarczyk (2013) observó que los factores mentales preconstruidos por los administradores e inversores presentan rigidez en las decisiones respecto a las necesidades de flexibilidad e innovación en los 
procesos, estando dispuestos a no asumir los riesgos en periodos de crisis. Kaliani et al. (2016) emplean un marco recientemente desarrollado para examinar la compleja relación del rendimiento entre los diferentes actores de la cadena de suministro. Las investigaciones concernientes a las relaciones de las variables de estudio en las pymes, específicamente del SLP, no existen, por lo que, al no tener mucha información sobre los análisis del rendimiento en las decisiones administrativas y en la GC, así como tampoco, sobre los procesos necesarios de la CEI, se considera importante este estudio (Vieera, 2014).

Por lo tanto, los modelos teóricos existentes acerca del conocimiento e innovación son conceptuales y diferencian sus aplicaciones por (1) el tamaño de la empresa, (2) la clase de servicio, (3) los factores humanos, (4) los factores psicológicos, y (5) los procesos que realizan las organizaciones (Firdauz et al., 2015). En el enunciado se muestra cómo inciden las variables de GC y CEI en las pymes del SLP y la dificultad de estas organizaciones para mantenerse en el mercado portuario. Esto es, que las pymes, en su mayoría, adolecen de (a) una gestión estructurada del conocimiento en sus organizaciones, (b) capacidad ejecutiva para innovar, y (c) un buen rendimiento en toda la cadena de suministro.

\section{Rendimiento en la cadena de suministros (RCS)}

Al-Hakim y Hassan (2013) indican que el rendimiento consiste en comparar los resultados esperados con los reales, definiéndolo como la evaluación del desempeño individual y organizacional, examinando el progreso del cumplimiento de los objetivos específicos. De estas afirmaciones se puede acotar que los indicadores del rendimiento brindan a los ejecutivos herramientas para mantener la posición competitiva de la organización (Fantazy y Salem, 2016). El RCS permite observar el comportamiento de los distintos procesos dentro de la CS, sus etapas o fases en la operación de la organización; consideran que dentro de las organizaciones el rendimiento está en los procesos entrópicos que miden las entradas y salidas del conocimiento de forma discreta apropiándose del mismo; usualmente los procesos se establecen según las metas cuantificables para los sistemas primarios, estos luego son asociados a las pequeñas metas y a los logros de los componentes individuales de las funciones asociadas, a la CS.

La eficacia es el parámetro que recoge el cumplimiento de la tarea con los recursos asignados, tanto en tiempo como en costos de operación, pero no busca optimizarlos; por otro lado, la eficiencia se encarga de realizar la tarea de manera óptima, aprovechando el método adecuado y en el menor tiempo posible en coordinación con los departamentos de proveedores y ventas; de esta manera, el rendimiento permite analizar los beneficios tangibles e intangibles en el intercambio de la eficiencia y la eficacia (Collins et al., 2010). Kaliani et al. (2016) presentan constructos e indicadores de las mejores prácticas en la CS, uno de los constructos es el RCS, valorando cinco indicadores, tres de los cuales fueron incorporados al constructo de eficacia, debido a su aporte en el funcionamiento interno de las Pymes y por temas de manejabilidad del conjunto de indicadores y disponibilidad de la información requerida dentro de la empresa, descartando así el indicador de coordinación con los clientes, al asumirlo como un indicador externo no administrado por la organización. El modelo para esta variable se lo diseñó, tal como se muestra en la Fig. 1.

\section{Gestión del conocimiento (GC)}

La necesidad de medir a la GC se fundamenta en las mismas capacidades de las organizaciones en ayudar a facilitar y mejorar las actividades de la GC por medio del análisis de sus factores, sin embargo, la GC debe implementar procesos formales que reflejen su rendimiento mediante la teoría del conocimiento basada en los recursos, en esta teoría, los recursos son todos los conocimientos tácitos y explícitos, internos y externos que son propiedad de las organizaciones (Cheng y Kuan, 2015). Adaptar la GC dentro de las organizaciones para obtener un RCS óptimo indispone, por lo general, a los miembros de las mismas. Esto se debe a las diferencias sustanciales que surgen en la adquisición y dinámica del conocimiento y su aplicación dentro de los procesos, en especial, cuando los encargados no poseen una idea concreta de los distintos sectores de las cadenas de suministros portuarias (Lambe, 2011).

De Gooijer (2000) y Beijerse (2000) coinciden que para la GC, como factor orientado a los recursos de las organizaciones, se utilizan constructos tales como (a) cultura, (b) estrategias, (c) infraestructura organizacional, (d) recursos; por su parte, Cheng y Kuan (2015) en su estudio acerca del desarrollo y validación de las mediciones del rendimiento de la GC para las pymes, presenta a la variable en 14 constructos, sin embargo, esta investigación está enfocada en la GC orientada a los recursos que las pymes tienen dentro de su organización, debido a las características presentes en las pymes SLP del Ecuador, tal como se muestra en la Fig. 2. De esta forma, se representa a la GC como un modelo integrado en las pymes donde los procesos están alineados a los objetivos organizacionales, permitiendo a las empresas una integración completa de sus capacidades y obteniendo un conocimiento productivo enfocado en el desarrollo del aprendizaje en las organizaciones. 


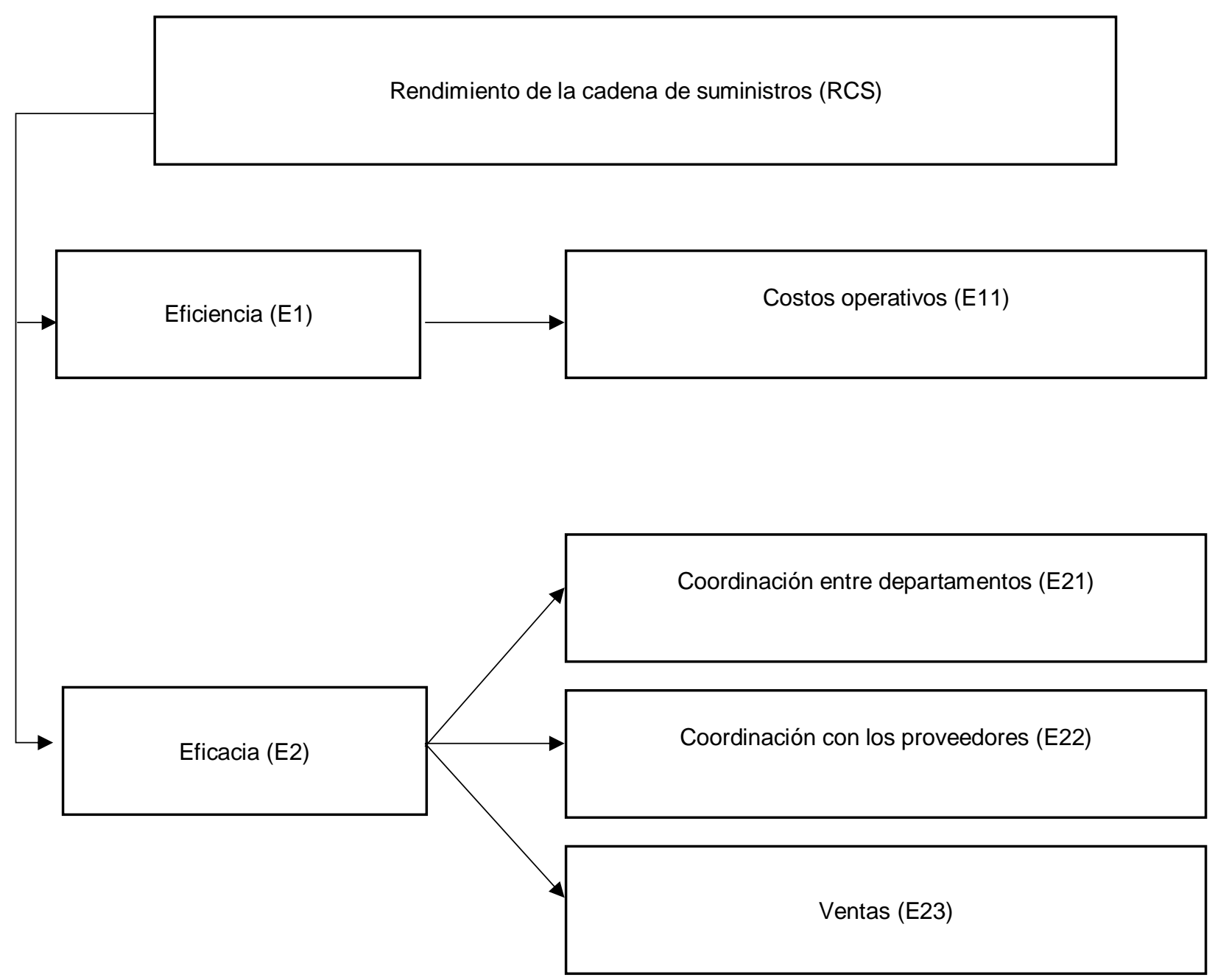

Fig. 1: Rendimiento de la cadena de suministros

\section{Capacidad ejecutiva para la innovación (CEI)}

La CEI explota las competencias para mejorar la competitividad en las pymes; sin embargo, Kearney et al. (2018) sostienen que se encuentran problemas (1) cuando se vincula la capacidad de gestión y la innovación, y (2) cuando la administración de las pymes mantiene un exceso de habilidades analíticas y descuidan los problemas que contemplan soluciones y toma de decisiones. En este sentido, en la CEI están presentes tres componentes basados en la teoría de las capacidades dinámicas (vea la Fig. 3) los cuales son (a) la capacidad organizacional, (b) la revitalización de las estrategias, y (c) el mejoramiento continuo (Oliva et al., 2019). En virtud de ello, la finalidad de la $\mathrm{CEI}$, es aplicar las últimas tecnologías, que cada vez son más aceleradas, tendiendo a la innovación y adaptación con una predisposición de mejora continua de los procesos y de la reducción de costos. El desarrollo de CEI redefine los modelos de negocios en la que se requieren capacidades de detección de los cambios ambientales basados en la comprensión de la naturaleza con nuevas posibilidades para la generación de valor de las empresas (Cooper et al., 2016).

\section{Hipótesis de estudio}

Las hipótesis por evaluarse asumen que la variable de la GC está dirigida hacia el comportamiento grupal de los sujetos cuando interviene la CEI, esta intervención también permite medir el RCS en las pymes y por tal comparar la relación GC y RCS anterior a la aplicación de la CEI y la relación GC-CEl y RCS, en este sentido, las organizaciones del sector portuario admiten el desarrollo y desempeño de sus procesos en las cadenas de suministros, sin embargo, la mayoría de las pymes de este sector desarrollan sus actividades, muchas de ellas, de manera empírica o ajustan sus actividades de acuerdo a la necesidad del sistema, pero no llevan un registro de esos conocimientos lo que les impide medir realmente el rendimiento de sus innovaciones dentro de la CS, además que muchos de estos conocimientos se pierden porque no son compartidos entre el personal de manera explícita, ante la inexistencia de evidencias en la literatura, relacionadas a las pymes del sector portuario, se plantean las siguientes cuatro hipótesis: dos relacionadas con el efecto positivo la GC y dos sobre el efecto positivo en la CEI. 


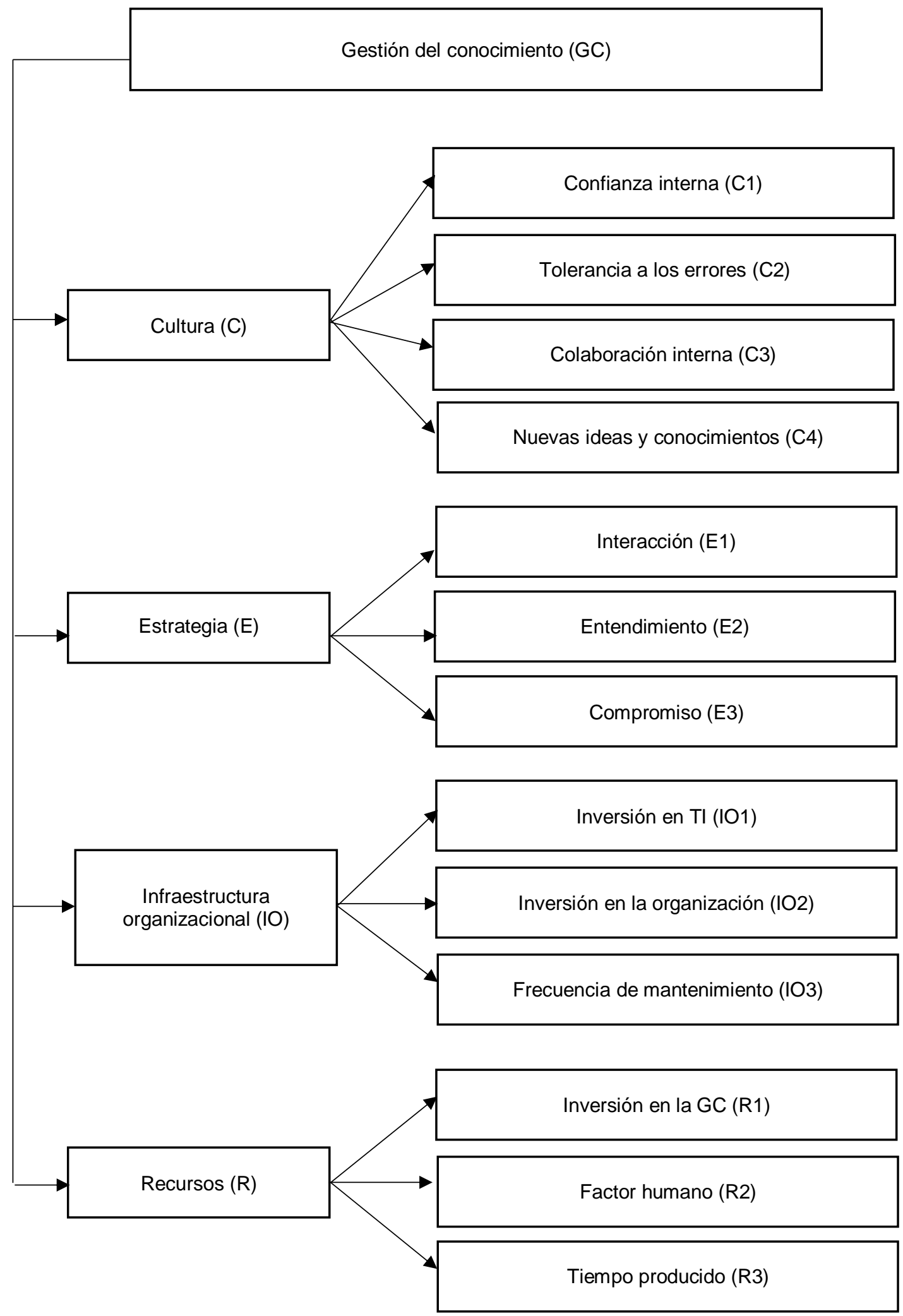

Fig. 2: Gestión del conocimiento basado en la teoría de los recursos 


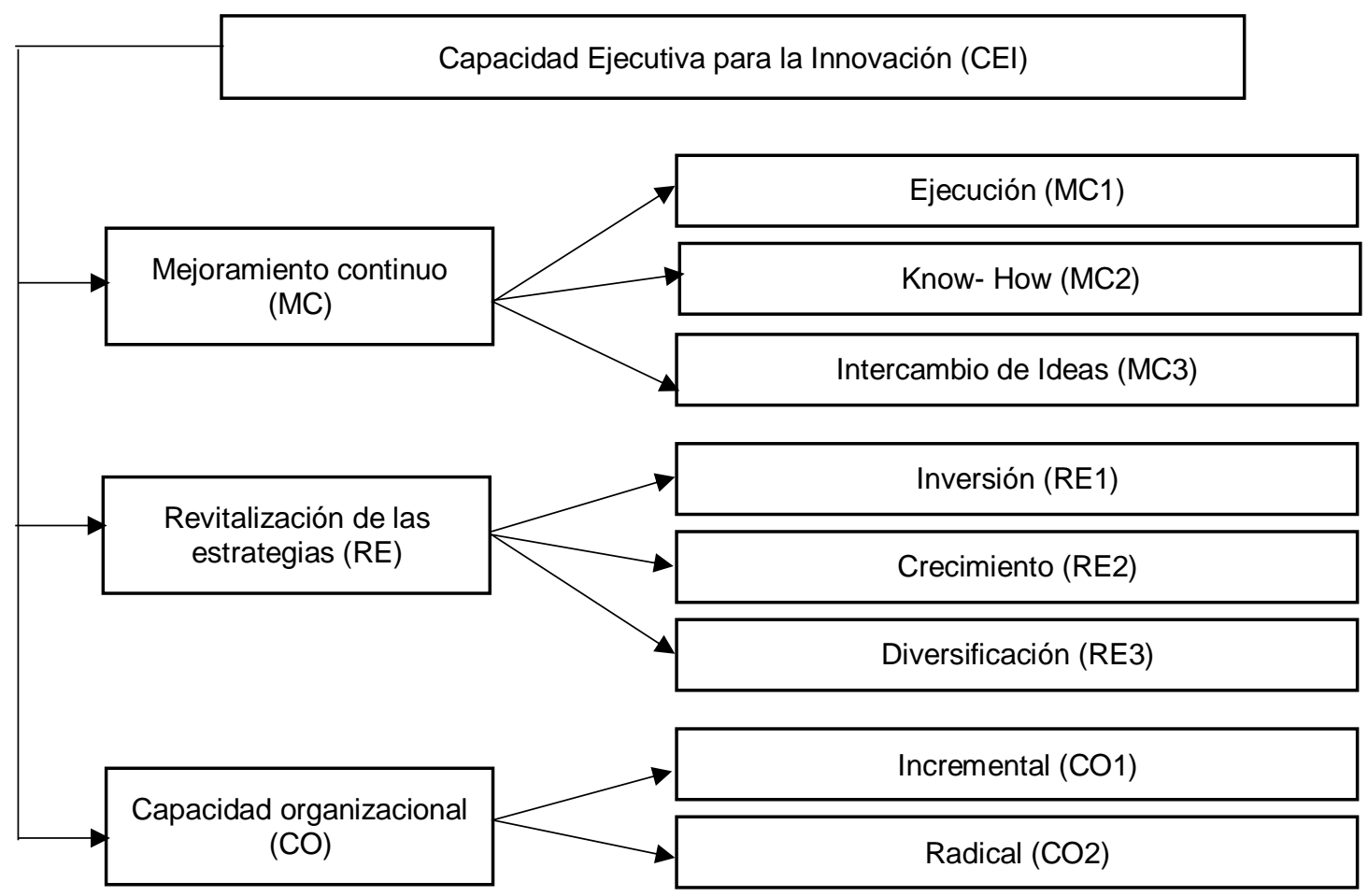

Fig. 3: Capacidad Ejecutiva para la Innovación orientado a las pymes

H1: La GC ejerce un efecto positivo en el RCS: Al-Hakim y Hassan (2013) manifestaron que la GC influye positivamente en el rendimiento organizacional, consideraron que la GC mejora el rendimiento organizacional debido a que permite aprovechar los activos intangibles de la compañía. Se enfatiza que el desarrollo de políticas de GC, procedimientos y sistemas de manejo del conocimiento en la CS disminuye las brechas de comunicación, asegurando el uso práctico del conocimiento en las áreas críticas de trabajo en las organizaciones, la confianza en los equipos de trabajo, dentro de los factores críticos de adaptación de la CS a la GC; sin embargo, la estandarización de los procesos puede ser llamativo para que el personal obtenga experiencia, pero debe tenerse en cuenta el tipo de organización en que se implemente, siendo estas conscientes de la reciprocidad en la interdependencia de los niveles operativos, pudiendo existir un fracaso en la implementación de las medidas afectando el balance entre innovación y eficiencia requeridos por el mercado. Cheng y Kuan (2015) indican que los factores, los recursos y los procesos de la gestión del conocimiento son necesarios para un mejor entendimiento de las estrategias corporativas con la finalidad de evaluar el rendimiento.

H2: La GC tiene un efecto positivo en la CEl: Beijerse (2000) considera que la flexibilización es vital para determinar la brecha y compartir el conocimiento a nivel operacional. Mientras, Attia y Salama (2018) examinan el efecto de las capacidades del rendimiento organizacional, manifiestan que la capacidad para la innovación debe definirse en la habilidad de transferir el conocimiento e ideas en nuevos productos, procesos y sistemas para el beneficio de las firmas, y los accionistas sostienen que es un auto-reflejo de la organización y sus productos. Si el conocimiento es reconocido entonces es procesable, por tal la innovación de las firmas se logra a través de una integración de todos los contenidos de conocimiento, construyendo nuevos contenidos de conocimiento y fomentando la intercomunicación entre los equipos. Los sistemas de innovación son herramientas que identifican demandas como un método de transferencia, ya sea de operaciones o de la evolución de los proyectos.

H3: La CEI tiene un efecto positivo en el RCS: Kearney et al. (2018) destacan que la gestión es aprovechada por los ejecutivos acorde a las formas de innovaciones. Mientras Kwak et al. (2018) sugieren la necesidad de desarrollar capacidades, esforzándose y fomentando innovación. Asimismo, Fantazy y Salem (2016) indican que el potencial éxito en la CS está en la aplicación de estrategias que conduzcan al rendimiento en todos los ámbitos operativos, aprovechando su eficiencia y eficacia. La capacidad de innovar de las firmas depende de los recursos que estas tengan, indicando que las empresas locales tienden a no tener un rendimiento adecuado incluso teniendo los recursos tecnológicos y las capacidades de innovar. 
H4: La CEl tiene un efecto mediador entre la GC y el RCS: El proceso de innovación debe tener alta colaboración por parte de los ejecutivos mediante el uso correcto de la GC en la adaptación de sus factores. También Attia y Salama (2018) señalan que compartir información y cooperación facilitaría la creación de nuevos procesos y productos. Entre tanto, Al-Hakim y Hassan (2013) examinan las relaciones de la GC y la CEI las cuales tienen efectos positivos en el rendimiento a través de los efectos de mediación de la innovación. De esta forma, Oliva et al. (2019) determinan que la GC y la CEI contribuyen a la administración por objetivos. La Fig. 4 muestra las relaciones entre las variables de estudio que se plantean en las hipótesis de investigación.

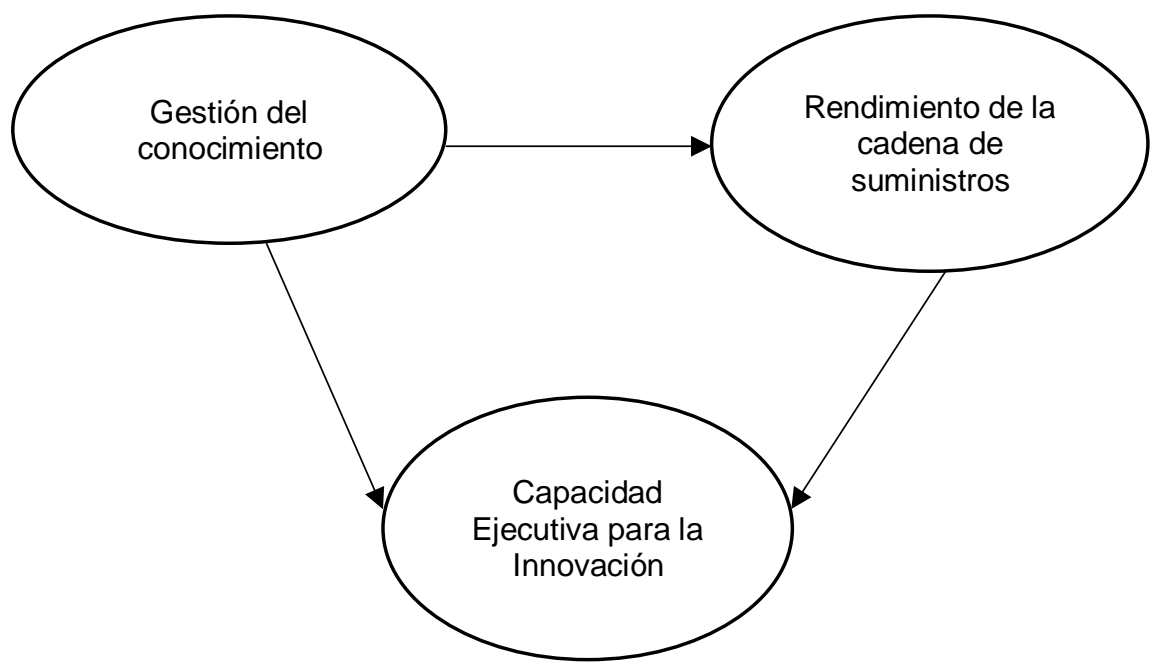

Fig. 4: Relación entre las variables de GC, CEl y RCS

\section{METODOLOGÍA}

Para comprobar las hipótesis de investigación se tomó como instrumento de recolección de datos dos cuestionarios y un marco teórico de autores que estudiaron las variables que se proponen en este estudio, estos son: para medir la GC se adaptaron los instrumentos desarrollados por Beijesrse, (2000), De Gooijer (2000) y Cheng y Kuan (2015); para la CEl se tomaron los constructos del marco teórico desarrollado por Kearney et al. (2018) acerca de las capacidades dinámicas, adaptándolo al cuestionario elaborado por Martínez et al., (2008) en la escala de medición y forma de redacción de los ítems; y finalmente, para el RCS se adaptaron los instrumentos desarrollados por Kaliani et al. (2016) a la necesidad de estudio de la realidad ecuatoriana de las Pymes. Se utilizó el método de la doble traducción para verificar que los cuestionarios mantengan el mismo sentido de los instrumentos originales. Cada uno de los cuestionarios fue validado por un panel de ocho expertos, quienes calificaron el sentido, la pertinencia y la redacción de cada pregunta. Algunos ítems fueron modificados para poder contextualizarlos a la realidad ecuatoriana.

Por último, se realizaron dos pruebas piloto que permitieron afinar detalles referentes a la claridad de la redacción para mejorar comprensión lectora de los encuestados con respecto al planteamiento de las preguntas y así evitar sesgos en la obtención de respuestas. Esta investigación es de enfoque cuantitativo, de lógica deductiva, de diseño no experimental, de tipo transversal y de carácter explicativo correlacional. La técnica estadística que se utilizó para comprobar las hipótesis planteadas fueron las ecuaciones estructurales, estimando los parámetros a través de la función de máxima verosimilitud.

\section{Población y muestra}

La población de estudio fueron las Pymes del SLP del Ecuador, misma que se determinó mediante la desagregación de una base de datos, con corte a septiembre de 2019 de la Superintendencia de Compañías, Valores y Seguros (SCVyS). El manejo de esta base de datos consistió en seleccionar a las provincias con mayor concentración de empresas portuarias o con relación directa a los puertos, aeropuertos y depósitos comerciales u otras empresas relacionadas al SLP, siendo las provincias de la región costa las que tienen la mayor cantidad de empresas del sector de estudio, adicional se utilizaron bases de datos del Instituto Nacional de Estadísticas y Censos (INEC) para hacer comparaciones. La región costa presenta el mayor número de empresas con un $51.29 \%$ del total, siendo aproximadamente 34075 empresas, por otro lado, las provincias que la componen, es decir, Esmeraldas, Manabí, Guayas, Los Ríos, El Oro y Santa Elena representan el $1.05 \%, 7.95 \%, 80.10 \%, 2.61 \%, 6.51 \%$ y $1.78 \%$ respectivamente. 
No obstante, las Pymes en esta región suman únicamente 12565. Posterior, se llevó a cabo un filtro, según la Clasificación Nacional de Actividades Económicas (CIIU REV. 4.0) basado en la Clasificación Internacional Industrial Uniforme (CIIU), donde el criterio de selección fue "las actividades económicas", discriminando en relación a las Pymes que están operando en el SLP, lo cual se presenta en la Tabla 1, cabe mencionar que la caracterización buscada de las empresas cumple con los parámetros establecidos según la definición dada por la CAN, esto es que (a) están operativas, (b) legalmente constituidas, (c) registradas como contribuyentes, (d) llevan registros contables, y (e) pertenecen al sector privado, misma que se siguió para este estudio. La Tabla 2 presenta el número de empresas del SLP por provincias, se observa, la provincia de Los Ríos fue excluida en circunstancia de no tener terminales portuarios por ser meridional; así, la población de estudio está distribuida de la siguiente manera.

Tabla 1: Número de empresas según su actividad económica relacionada con el SLP

\begin{tabular}{|c|c|c|c|c|}
\hline CIIU & Actividad económica & Pequeña & Mediana & Total \\
\hline H4923.01 & Actividades de transporte de carga por carretera. & 624 & 121 & 745 \\
\hline H4923.02 & Servicio de alquiler de camiones. & 2 & 0 & 2 \\
\hline H4923.05 & Otros servicios de transporte regular o no de carga por carretera. & 0 & 0 & 0 \\
\hline H5012.01 & Transporte marítimo y de cabotaje, regular o no regular, de carga. & 27 & 11 & 38 \\
\hline H5012.02 & Transporte de barcazas, plataformas petrolíferas, etc. & 3 & 0 & 3 \\
\hline H5012.03 & $\begin{array}{l}\text { Alquiler de embarcaciones con tripulación para el transporte } \\
\text { marítimo de mercancías. }\end{array}$ & 0 & 1 & 1 \\
\hline H5022.01 & $\begin{array}{l}\text { Transporte de carga por ríos y otras vías de navegación interiores, } \\
\text { incluidos puertos interiores. }\end{array}$ & 7 & 1 & 8 \\
\hline H5210.00 & $\begin{array}{l}\text { Actividades de almacenamiento y depósito para todo tipo de } \\
\text { productos. }\end{array}$ & 12 & 8 & 20 \\
\hline H5222.00 & $\begin{array}{l}\text { Actividades relacionadas con el transporte acuático de pasajeros, } \\
\text { animales o carga. }\end{array}$ & 17 & 5 & 22 \\
\hline H5224.00 & Carga y descarga de mercancías y equipaje, estiba y desestiba. & 53 & 20 & 73 \\
\hline H5229.01 & $\begin{array}{l}\text { Servicios transitorios de organización o coordinación de operaciones } \\
\text { de transporte y flete por tierra, mar y aire. }\end{array}$ & 12 & 5 & 17 \\
\hline H5229.02 & $\begin{array}{l}\text { Actividades logísticas: planificación, diseño y apoyo de operaciones } \\
\text { de transporte, almacenamiento y distribución, etc. }\end{array}$ & 41 & 22 & 63 \\
\hline H5229.03 & $\begin{array}{l}\text { Actividades de agentes de aduanas, emisión y tramitación de } \\
\text { documentos de transporte y conocimientos de embarque. }\end{array}$ & 42 & 8 & 50 \\
\hline
\end{tabular}

Tabla 2: Población de estudio dividida por provincias

\begin{tabular}{|l|c|}
\hline \multicolumn{1}{|c|}{ Provincias } & $N^{\circ}$ de Pymes \\
\hline El Oro & 98 \\
\hline Esmeraldas & 17 \\
\hline Manabí & 113 \\
\hline Guayas & 735 \\
\hline Santa Elena & 25 \\
\hline Total & 988 \\
\hline
\end{tabular}


La técnica de muestreo utilizada fue un muestreo aleatorio simple, el marco muestral lo constituyó la lista de 988 Pymes de las cinco provincias de la costa ecuatoriana que realizan actividades económicas relacionadas al sector portuario según la CIIU REV. 4.0. Para conocer el tamaño de la muestra de estudio, se empleó un error muestral del $5 \%$, un nivel de confianza del $95 \%$ y una probabilidad de éxito igual a la probabilidad de fracaso, en consecuencia, la muestra representativa es de 277 pymes del SLP ubicadas en las provincias de la región costa del Ecuador con excepción de la provincia de Los Ríos.

\section{RESULTADOS Y DISCUSION}

Esta sección se encuentra divida en dos subsecciones: una describe el modelo de medición y la otra explica el modelo estructural detrás de las variables y el respectivo testeo de hipótesis de investigación para determinar si existe la mediación propuesta en las hipótesis.

\section{Modelo de medición}

Antes de realizar la estimación de los parámetros a través de la función de máxima verosimilitud de las ecuaciones estructurales, se procedió a realizar la verificación del modelo de medida de cada uno de los constructos del modelo. Se realizó un análisis factorial confirmatorio en cada una de las variables: GC, CEI y RCS para verificar tanto la validez convergente de los ítems, la validez discriminante entre las diferentes dimensiones de los constructos, así como la confiabilidad de las mediciones. A continuación, en la Tabla 3, se presentan los resultados. Así mismo, se verificó el cumplimiento del supuesto de normalidad multivariante a través del coeficiente de Mardia, cuyo valor fue de 24,0581. Rodríguez y Ruiz (2008) señalaron que, si el coeficiente de Mardia es menor que 50, los parámetros estimados a través de la función de máxima verosimilitud son consistentes; siempre y cuando se cuente con un tamaño muestral adecuado y un modelo bien especificado.

La validez convergente se evaluó mediante el cumplimiento de tres criterios: (a) que los factores de carga sean significativos; (b) que el factor de carga sea mayor que $0.5 ; \mathrm{y}$, (c) que el promedio extraído de la varianza (AVE) sea mayor que 0.5 (Hair et al., 1999). Tal como se puede observar en la Tabla 3, vemos que todos los ítems excepto EST002, son mayores que 0.5. El promedio extraído de la varianza de cada constructo es mayor que 0.5 , lo que se interpreta como que los ítems explican más del $50 \%$ de la varianza del constructo. Finalmente, todos los factores de carga resultaron significativamente diferentes de cero, excepto EST002, por lo que se decidió separar este ítem del análisis subsiguiente.

Tabla 3: Validez convergente y confiabilidad compuesta

\begin{tabular}{|c|c|c|c|}
\hline Constructo & Factor de Carga & $C R$ & $A V E$ \\
\hline \multicolumn{2}{|c|}{ Infraestructura Organizacional (INF) } & 0.89 & 0.53 \\
\hline INF001 & 0.855 & & \\
\hline INF002 & 0.943 & & \\
\hline INF003 & 0.765 & & \\
\hline INF004 & 0.806 & & \\
\hline \multicolumn{2}{|c|}{ Estrategia (EST) } & 0.82 & 0.61 \\
\hline EST001 & 0.677 & & \\
\hline EST002 & 0.154 & & \\
\hline EST003 & 0.811 & & \\
\hline EST004 & 0.792 & & \\
\hline EST005 & 0.799 & & \\
\hline \multicolumn{2}{|c|}{ Recursos (REC) } & 0.76 & 0.57 \\
\hline REC001 & 0.544 & & \\
\hline REC002 & 0.596 & & \\
\hline REC003 & 0.777 & & \\
\hline REC004 & 0.502 & & \\
\hline
\end{tabular}


Tabla 3 (Continuación)

\begin{tabular}{|c|c|c|c|}
\hline Constructo & Factor de Carga & $C R$ & AVE \\
\hline \multicolumn{2}{|c|}{ Cultura (CULT) } & 0.93 & 0.72 \\
\hline CULT001 & 0.713 & & \\
\hline CULT002 & 0.846 & & \\
\hline CULT003 & 0.893 & & \\
\hline CULT004 & 0.901 & & \\
\hline CULT005 & 0.876 & & \\
\hline \multicolumn{2}{|c|}{ Capacidad Organizacional (CO) } & 0.95 & 0.64 \\
\hline $\mathrm{CO001}$ & 0.866 & & \\
\hline CO002 & 0.769 & & \\
\hline CO003 & 0.849 & & \\
\hline CO004 & 0.767 & & \\
\hline \multicolumn{2}{|c|}{ Revitalización de Estrategias (RE) } & 0.77 & 0.58 \\
\hline RE001 & 0.599 & & \\
\hline RE002 & 0.673 & & \\
\hline RE003 & 0.610 & & \\
\hline RE004 & 0.842 & & \\
\hline RE005 & 0.704 & & \\
\hline \multicolumn{2}{|c|}{ Mejoramiento Continuo (MC) } & 0.84 & 0.63 \\
\hline MC001 & 0.798 & & \\
\hline MC002 & 0.622 & & \\
\hline MC003 & 0.834 & & \\
\hline MC004 & 0.198 & & \\
\hline \multicolumn{2}{|c|}{ Eficiencia (ERCS) } & 0.96 & 0.65 \\
\hline EFF001 & 0.846 & & \\
\hline EFF002 & 0.792 & & \\
\hline EFF003 & 0.904 & & \\
\hline EFF004 & 0.866 & & \\
\hline \multicolumn{2}{|c|}{ Eficacia (EFRCS) } & 0.91 & 0.69 \\
\hline Constructo & Factor de Carga & $C R$ & AVE \\
\hline EFZ001 & 0.622 & & \\
\hline EFZ002 & 0.876 & & \\
\hline EFZ003 & 0.943 & & \\
\hline EFZ004 & 0.734 & & \\
\hline
\end{tabular}

Tal como recomendaron Chión y Vincent (2016) la validez discriminante se evaluó a través de un análisis correlacional entre todos los constructos de primer orden, tanto dependientes como independientes, en un solo gráfico de AMOS SPSS. Se planteó como hipótesis alternativa que la correlación entre el constructo a y el constructo $b$ no era igual a uno y como hipótesis nula que dicha correlación era igual a 1 ; y así, por cada par de constructos. 
$H_{0}: \varphi_{I N F-E S T}=1$

$H_{1}: \varphi_{I N F-E S T} N F-$

Donde $t_{\text {calculado }}=\left(\varphi_{\text {INF-EST }}-1\right) / \sigma_{\text {INF-EST }}$ y donde $t_{\text {crítico }}$ es dado por la tabla $t$ para una prueba de dos colas con un nivel de significancia ( $\alpha$ ) de $5 \%$ con 466 grados de libertad. En la Tabla 4 se presentan los resultados del análisis de las correlaciones entre todos los constructos.

Tabla 4: Validez discriminante

\begin{tabular}{|c|c|c|c|c|c|c|}
\hline \multicolumn{3}{|c|}{ Correlación } & Estimado & Desv. est. & Tprueba & Significancia \\
\hline INF & $<-->$ & EST & 0.744 & 0.03 & -8.258 & $* * *$ \\
\hline INF & $<-->$ & REC & 0.638 & 0.02 & -15.739 & $* * *$ \\
\hline INF & $<-->$ & CULT & 0.867 & 0.02 & -8.867 & $* * *$ \\
\hline INF & $<-->$ & $\mathrm{CO}$ & 0.140 & 0.02 & -45.263 & $* * *$ \\
\hline INF & $<-->$ & RE & -1.246 & 0.02 & -93.583 & $* * *$ \\
\hline INF & $<-->$ & MC & 1.721 & 0.03 & 23.258 & $* * *$ \\
\hline INF & $<-->$ & ERCS & 0.269 & 0.03 & -22.844 & $* * *$ \\
\hline INF & $<-->$ & EFRCS & 2.741 & 0.02 & 96.722 & $* * *$ \\
\hline EST & $<-->$ & REC & -0.619 & 0.01 & -134.917 & $* \star *$ \\
\hline EST & $<-->$ & CULT & -0.217 & 0.02 & -67.611 & $* * *$ \\
\hline EST & $<-->$ & $\mathrm{CO}$ & 0.219 & 0.02 & -41.105 & $* * *$ \\
\hline EST & $<-->$ & RE & 0.853 & 0.03 & -5.444 & $* * *$ \\
\hline EST & $<-->$ & MC & 0.682 & 0.03 & -12.720 & $* * *$ \\
\hline EST & $<-->$ & ERCS & -0.485 & 0.01 & -247.500 & $* * *$ \\
\hline EST & $<-->$ & EFRCS & 1.277 & 0.01 & 30.778 & $* * *$ \\
\hline REC & $<-->$ & CULT & 0.458 & 0.01 & -41.692 & $* * *$ \\
\hline REC & $<-->$ & $\mathrm{CO}$ & 0.438 & 0.02 & -26.762 & $* * *$ \\
\hline REC & $<-->$ & $\mathrm{RE}$ & 0.764 & 0.02 & -11.800 & $* * *$ \\
\hline REC & $<-->$ & MC & 0.509 & 0.01 & -44.636 & $* * *$ \\
\hline REC & $<-->$ & ERCS & -0.061 & 0.01 & -96.455 & $* * *$ \\
\hline REC & $<-->$ & EFRCS & -0.111 & 0.02 & -69.438 & $* * *$ \\
\hline CULT & $<-->$ & $\mathrm{CO}$ & -0.791 & 0.01 & -137.769 & $* * *$ \\
\hline CULT & $<-->$ & RE & -1.237 & 0.02 & -149.133 & $* * *$ \\
\hline CULT & $<-->$ & MC & -0.731 & 0.02 & -86.550 & $* * *$ \\
\hline CULT & $<-->$ & ERCS & -0.554 & 0.02 & -81.790 & $* * *$ \\
\hline
\end{tabular}


Tabla 4 (Continuación)

\begin{tabular}{|c|c|c|c|c|c|c|}
\hline \multicolumn{3}{|c|}{ Correlación } & Estimado & Desv. est. & Tprueba & Significancia \\
\hline CULT & $<-->$ & EFRCS & 0.398 & 0.02 & -28.667 & $* * *$ \\
\hline $\mathrm{CO}$ & $<-->$ & RE & 0.497 & 0.02 & -22.864 & $* * *$ \\
\hline $\mathrm{CO}$ & $<-->$ & MC & 0.135 & 0.03 & -27.903 & $* * *$ \\
\hline $\mathrm{CO}$ & $<-->$ & ERCS & -0.546 & 0.02 & -96.625 & $* * *$ \\
\hline $\mathrm{CO}$ & $<-->$ & EFRCS & 0.843 & 0.03 & -4.618 & $* * *$ \\
\hline RE & $<-->$ & MC & 0.599 & 0.01 & -28.643 & $* * *$ \\
\hline $\mathrm{RE}$ & $<-->$ & ERCS & -0.746 & 0.01 & -194.000 & $* * *$ \\
\hline RE & $<-->$ & EFRCS & 0.671 & 0.02 & -19.353 & $* * *$ \\
\hline $\mathrm{MC}$ & $<-->$ & ERCS & 0.711 & 0.01 & -22.231 & $* * *$ \\
\hline$M C$ & $<-->$ & EFRCS & 0.693 & 0.02 & -14.619 & $* * *$ \\
\hline ERCS & $<-->$ & EFRCS & 0.715 & 0.01 & -25.909 & $* * *$ \\
\hline
\end{tabular}

Tal como se puede apreciar en la Tabla 4, todos los $t$ de prueba son mayores que 2, por lo que se rechazan las hipótesis nulas y se acepta que las correlaciones entre los constructos no son iguales a 1, evidenciando que no son los mismos constructos, sino que se diferencian entre sí. Cabe mencionar que, debido al alto número de grados de libertad, el estadístico $t$ se aproxima a la distribución normal. La confiabilidad de las mediciones se evaluó mediante dos criterios: (a) el índice de confiablidad compuesta ( $\rho^{2}$ ) y (b) los índices de bondad de ajuste de cada uno de los constructos: GC, CEI y RCS. Es importante recordar que, la variable GC es un constructo de segundo orden, compuesto por las dimensiones: infraestructura organizacional, estrategia, recursos y cultura; así mismo, la CEl, también es un constructo de segundo orden, conformado por la capacidad organizacional, revitalización de estratégicas y mejoramiento continuo. Finalmente, el RCS se forma por los constructos de primer orden eficiencia y eficacia. En la Tabla 5 se presentan los indicadores de bondad de ajuste de todas las variables de segundo orden propuestas en el modelo.

Tabla 5: Indicadores de bondad de ajuste

\begin{tabular}{|l|c|c|c|c|c|}
\hline \multirow{2}{*}{\multicolumn{1}{|c|}{ Constructo }} & \multicolumn{5}{c|}{ Indicadores } \\
\cline { 2 - 6 } & GFI & AGFI & CFI & TLI & RMSEA \\
\hline Gestión del Conocimiento & 0.96 & 0.89 & 0.97 & 0.97 & 0.06 \\
\hline Capacidad Ejecutiva de Innovación & 0.94 & 0.81 & 0.97 & 0.97 & 0.05 \\
\hline Rendimiento de la Cadena de Suministros & 0.92 & 0.86 & 0.96 & 0.98 & 0.05 \\
\hline
\end{tabular}

Tal como se puede observar, los indicadores de bondad de ajuste de cada uno de los constructos están dentro de los rangos de aceptación. Los coeficientes de confiabilidad compuesta $\left(\rho^{2}\right)$ son todos mayor que 0.7 , (Ver Tabla 3). Por todo lo expuesto, podemos concluir que las mediciones son confiables y válidas tanto convergentemente como discriminante.

\section{Modelo estructural y prueba de hipótesis}

El modelo estructural mide las relaciones entre los constructos. Con la finalidad de simplificar la presentación de los resultados, se presenta la Fig. 5, con los factores de carga estandarizados entre los constructos de segundo orden. Así, como con sus respectivas dimensiones. 


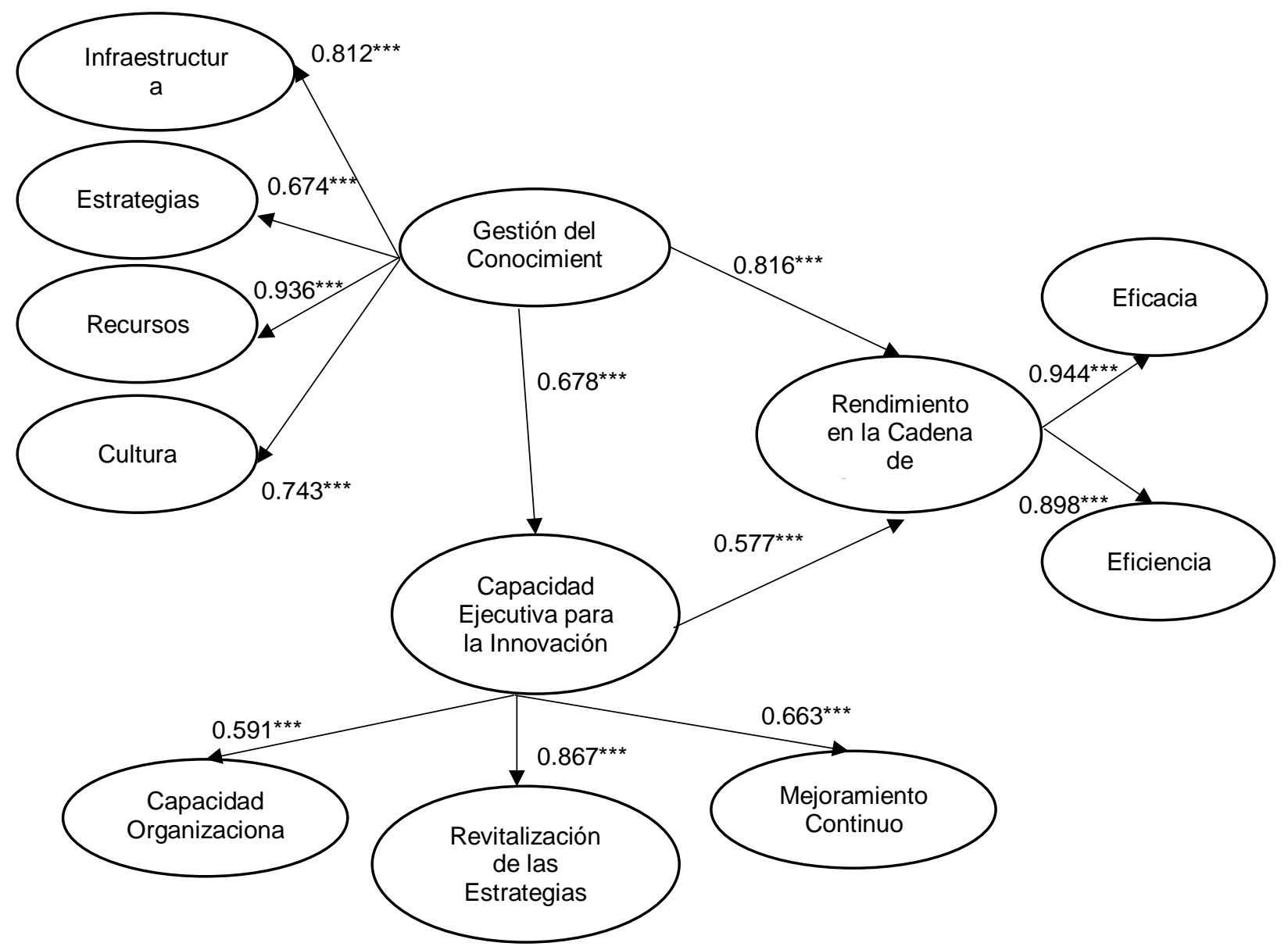

Fig. 5: Modelo estructural de la relación entre la capacidad ejecutiva de innovación la gestión del conocimiento y el rendimiento de la cadena de suministros.

El método de estimación de los parámetros de las relaciones entre los constructos se realizó a través del software SPSS AMOS 23, con la aplicación de la función de máxima verosimilitud. De los resultados sin estandarizar se verificó que todas las cargas son significativas $\left(^{* * *}\right)$, por lo que se pudieron comprobar todas las hipótesis planteadas. La relación entre la CEI y el RCS tiene un coeficiente de 0.577. Debido a que el signo del coeficiente es positivo, se interpreta que la relación que existe entre la CEI y el RCS es directa. Así mismo, la GC tiene un efecto significativo sobre la CEI. Es un efecto positivo, esto es, a mayor GC, mayor CEI. Por último, el efecto de la GC sobre el RCS también es un efecto positivo y significativo.

El modelo estructural es evaluado mediante los índices de bondad de ajuste, así tenemos que el GFI es de 0.885 , el AGFI es de 0.838, el TLI es de 0.902, el CFI es de 0.940, el RMSEA es de 0.08 . El R ${ }^{2}$ múltiple del RCS es de 0.62 , lo que quiere decir, que el modelo explica el $62 \%$ de la varianza de esta variable. Así mismo, el $\mathrm{R}^{2}$ múltiple de la CEl es de 0.44 , lo que quiere decir que el modelo explica el $44 \%$ de la variación de este constructo. La verificación del efecto mediador de la CEI entre la GC y el RCS se realizó mediante la prueba de Sobel (1986). El estadístico de Sobel se calculó en 3.78 con un valor $p$ de 0.000 por lo que se acepta la hipótesis de que el efecto indirecto que ejerce la GC a través de la CEI hacia el RCS es significativo. Por lo que se confirma la hipótesis de la mediación. La CEI es una mediadora parcial entre la GC y el RCS, debido a que tanto el efecto directo como el efecto indirecto de la GC sobre el RCS, son significativos. Debemos recordar, que el efecto indirecto de la GC sobre el RCS, se calcula multiplicando el efecto directo de GC sobre la variable mediadora CEI por el efecto directo entre la variable mediadora y la dependiente. En este caso el efecto indirecto estandarizado es 0.391 .

Para respaldar el resultado obtenido sobre la mediación de la CEI entre la GC y el RCS, se procedió a realizar, de manera adicional, lo recomendado por Ató y Vallejo (2011) quienes señalaron que para comprobar el efecto mediador se deben desarrollar tres modelos alternativos: (a) el modelo solamente con el efecto $X \rightarrow Y$; (b) el modelo solamente con el efecto mediado $X \rightarrow Z \rightarrow Y$; $y$, (c) la prueba del efecto indirecto $X \rightarrow Z \rightarrow Y$ junto al efecto directo, bajo dos condiciones: $X \rightarrow Y$ restringido a cero y $X \rightarrow Y$ sin restringir; para finalmente, evaluar la bondad de ajuste de cada uno de estas alternativas. Se pudo comprobar que el modelo "a" presentó un GFI de 0.821 , valor superior al umbral de aceptación; así mismo, el modelo "b" presentó un GFI de 0.833, superior 
al umbral de aceptación; y que, finalmente en el modelo "c", a través de la prueba $x^{2}(c)=x^{2}(a)-x^{2}(b)$, donde "a" representa los grados de libertad del modelo restringido, "b" representa los grados de libertad del modelo sin restringir y "c" es la diferencia entre "a" y "b", (en este caso, el valor de "c" siempre será uno, debido a que la diferencia es solo una restricción); no se encontraron diferencias significativas entre los modelos restringido y sin restringir, resultado que evidencia la presencia del efecto de mediación de la variable CEI, tal como se lo planteó en la hipótesis de investigación.

\section{DISCUSION FINAL}

De acuerdo con los resultados, los valores de variación entre las variables GC y RCS aumentan a medida que los valores varían con la variable mediadora, $\mathrm{CEI}$, al tener una relación de 0.826 entre GC y RCS permite determinar una alta correlación entre ellas, estos resultados confirman lo señalado por Al-Hakim y Hassan (2013), quienes indican que la relación entre la GC y CEI tiene un efecto positivo en el rendimiento de mediación de la innovación al determinar que se están complementando entre sí; en el mismo sentido, Oliva et al. (2019) señalan que es el resultado del manejo y concreción de los objetivos de cada nivel de jerarquía de la empresa. Por otro lado, la CEI permite desarrollar una adquisición selectiva de la búsqueda del conocimiento y del proceso de la GC para implementarla a la CS, y por otro deja que este RCS permita ser medido y evaluado en el tiempo. Por su parte, la correlación de CEI con RCS es menor por su relación indirecta, tal como sostienen Kwok y Kian (2015) que la GC y RCS están ligados en todas las jerarquías de la empresa, Chen y Kirsis (2007) sostienen la importancia de la cooperación y las relaciones a largo plazo entre los miembros de la cadena de suministros, lo que facilita la obtención de resultados en la creación e innovación de productos o servicios a largo plazo. También, Fantazy y Salem (2016) indican que el potencial de éxito en la CS está en función de la aplicación de estrategias que demanden el uso de los recursos, siendo la CEI caracterizada por dichas estrategias y el RCS, una acción a realizarse de manera indirecta, por el manejo indebido de los recursos tecnológicos y capacidad inherente de innovar de las organizaciones.

Esta investigación sugiere que la correlación que puede tener la GC como punto referencial para el marco teórico que las pymes puedan aplicar, al momento de establecer un modelo de ejecución para la innovación de la CS en situaciones inesperadas de los mercados, delimitando los parámetros de riesgo que el personal asociado pueda ingresar a proyectos en áreas de alta sensibilidad. Otro punto que se excluyó del estudio fue la implicación de los accionistas en los procesos que requieren una restructuración de la CS, así como nuevos esquemas de negociación disruptivos internacionales, esto porque al identificar la fragilidad a la que están expuestas las empresas que no se internacionalizan, fundamentalmente por decisiones que exponen a una tendencia de perder mercados locales a las pymes, con mayor frecuencia que las empresas que se arriesgaban, sin importar el tamaño, diferenciando solamente la capacidad que tienen estas para al corto o mediano plazo implementar sus negocios y para obtener la ganancias. Finalmente, se exhorta a seguir estudiando si la CEI puede tener efectos mediadores en la rentabilidad de los proyectos que las pymes puedan realizar en mediano plazo.

\section{CONCLUSIONES}

De acuerdo al trabajo presentado y a los resultados obtenidos, se pueden plantear las siguientes conclusiones principales:

1. La GC establecen patrones de comportamiento que influyen indirectamente al RCS, en todos los niveles de la organización. Por lo tanto, el rendimiento es mediado a través de la CEI, cuando procesos de la GC están involucrado en la cadena de suministro de manera directa en el proceso de innovación. Sin embargo, se requiere determinar si esta capacidad se mantiene al largo plazo, debido a la dispersión en los años de existencia de las empresas que se utilizaron para la encuesta.

2. Los resultados indican que, el efecto mediador que ejerce la CEI, en la relación de los procesos que pretenden utilizar la GC para obtener un mayor RCS en proyectos de innovación, permitirían en las pymes del SLP, administrar aquellas fuentes de conocimiento, procesarlas, e incorporarlas a sus procedimientos, y apropiándose de la información, para así aplicarla en sí misma, con propósito de obtener resultados aplicables y retroactivos en sus operaciones.

3. Es imperativo de que las pymes reconozcan y evalúen sus sistemas de GC, para evaluar los resultados de procesos en su CS innovación de procesos o productos, permitiendo ahondar, en los efectos directos que puedan resultar en RCS, debido a la aplicación de la mediación de CEl.

4. La CEI, en las organizaciones tienen un amplio concepto de aplicación en los proyectos que la gerencia pretenda realizar, eliminando las barreras ineficaces de realización de los proyectos, permitiendo la flexibilización del pensamiento crítico en las distintas etapas que el proyecto se encuentre. Siendo concordante los resultados, con lo manifestado por Kearney et al., (2018), de la necesidad que el sector 
portuario aplique un marco ejecutorial de la capacidad innovativa, que permita la competitividad de las organizaciones en sectores estratégicos económicos, que para efecto de esta investigación se usó la unidad pymes del SLP.

\section{REFERENCIAS}

Adegbuyi, P. y Uhomoibhi, J., Trends in the development of technology and engineering education in emerging economies, doi: 10.1108/17504970810900432, Multicultural Education and Technology Journal, 2(3), 132-139 (2008).

Agostini, L., Nosella, A. y Filippini, R., Does intellectual capital allow improving innovation performance? A quantitative analysis in the SME context, doi: 10.1108/JIC-05-2016-0056, Journal of Intellectual Capital, 18(2), 400-418 (2017).

Algorta, M. y Zeballos, F., Human resource and knowledge management: best practices identification, doi:/10.1108/13683041111184125, Measuring Business Excellence, 15(4), 71-80 (2011).

Al-Hakim, L. y Hassan, S., Knowledge management strategies, innovation, and organizational performance, doi: 10.1108/09727981311327767, Journal of Advances in Management Research, 10(1), 58-71 (2013).

An, X., Deng, H. y otros 2 autores, Knowledge management in supporting collaborative innovation community capacity building, doi: 10.1108/JKM-10-2013-0413, Journal of Knowledge Management, 18(3), 574-590 (2014).

Antai, I., Supply chain vs supply chain competition: a niche-based approach, doi: 10.1108/01409171111171500, Journal of Knowledge Management, 34 (10), 1107-1124 (2011).

Ató M. y Vallejo G. Los efectos de terceras variables en la investigación psicológica, Anales de Psicología, 27(2) 550-561 (2011).

Attia, A. y Salama, I., Knowledge management capability and supply chain management practices in the Saudi food industry, doi: 10.1108/BPMJ-01-2017-0001, Business Process Management Journal, 24(2), 459-477 (2018).

Åslund A. y Bäckström I, Management processes and management's role in customer value creation, doi: 10.1108/IJQSS11-2015-0074, International Journal of Quality and Service Sciences, 9(2), 1-30 (2017).

Beijerse, R., Knowledge management in small and medium-sized companies: Knowledge management for entrepreneurs, doi: 10.1108/13673270010372297, Journal of Knowledge Management, 4(2), 162-179 (2000).

Busse, C. y Wallenburg C., Innovation management of logistics service providers foundations, review and research agenda, doi: 10.1108/09600031111118558, International Journal of Physical Distribution \& Logistics Management, 41(2), 187-218 (2011).

Buestán, M., y Van-Landeghem, H., Implementation of S-DBR in four manufacturing SMEs: a research case study, doi: 10.1080/09537287.2015.1015060, Production Planning and Control, 26(13), 1110-1127 (2015).

Carrillo, J., An ex-post comparative analysis of SME formation in Brazil and Mexico: Towards a research agenda, doi: 10.1108/17468800710739225, International Journal of Emerging Markets, 144-165 (2007).

Cela, K., Sicilia, M. y Sanchez, S., Comparison of collaboration and performance in groups of learners assembled randomly comparison of collaboration and performance in groups of learners assembled randomly or based on learners' topic preferences, Journal of Educational Technology and Society, EISSN-1436-4522, 18(4), 287-298 (2015).

Chen, I. y Kirsis, A., A research framework of sustainable supply chain management: the role of relational capabilities in driving performance, doi: 10.1108/IJLM-11-2016-0265, International Journal of Logistics Management, 28(4), 1454-1478 (2017).

Cheng, L. y Kuan, W., Development and validation of knowledge management performance measurement constructs for small and medium enterprises, doi: 10.1108/JKM-10-2014-0398, Journal of Knowledge Management, 19(4), 711-734 (2015).

Chión, S. y Vincent, C. Analítica de datos para la modelación estructural, 317-319, Pearson Educación de Perú, Lima, Perú (2016).

Cooper, A., Huscroft, J. y otros 2 autores, Knowledge management for logistics service providers: The role of learning culture, doi: 10.1108/IMDS-06-2015-0262, Industrial Management y Data Systems, 116(3), 1-34 (2016).

Collins, J., Worthington, W. y otros 2 autores, Knowledge management, supply chain technologies, and firm performance, doi: 10.1108/01409171011083969, Management Research Review, 33(10), 947-960 (2010).

Dahash, F., Examining the factors influencing knowledge management system (KMS) adoption in small and medium enterprises SMEs, doi: 10.1108/BPMJ-10-2016-0221, Business Process Management Journal, 24(1), $234-265$ (2017).

Darroch, J., Knowledge management, innovation and firm performance, doi: 10.1108/13673270510602809, Journal of Knowledge Management, 9(3), 101-115 (2005).

Davis, N., Ferding, R. y otros 3 autores, Innovative technologies for multicultural education needs, doi: 10.1108/17504970710745201, Multicultural Education and Technology Journal, 1(1), 47-63 (2007).

De Gooijer, J., Designing a knowledge management performance framework, doi: 10.1108/13673270010379858 (2000), Journal of Knowledge Management, 4(4), 303-310 (2000). 
Ding, Z., Jiang, S. y otros 2 autores, A new TRIZ-based patent knowledge management system for construction technology innovation, doi: 10.1108/JEDT-03-2016-0017, Journal of Engineering, Design and Technology, 1-17 (2017).

Echeverri, A., Lozada, N. y Arias, J.E., Incidencia de las Prácticas de Gestión del Conocimiento sobre la Creatividad Organizacional, doi: 10.4067/S0718-07642018000100071, Información Tecnológica, 29(1), 71-82 (2018).

El Baz, J., Laguir, I. y Stekelorum, R., Logistics and supply chain management research in Africa: A systematic literature review and research agenda, doi: 10.1108/IJLM-09-2017-0242, International Journal of Logistics Management, 30(1), 838 (2019).

Esmer, S., Nguyen, H. y otros 2 autores, K., Non-price competition in the port sector: A case study of ports in Turkey, doi:10.1016/j.ajsl.2016.03.001, Asian Journal of Shipping and Logistics, 32(1), 3-11 (2016).

Esper, T., Defee, C. y Mentzer, J., A framework of supply chain orientation, doi: 10.1108/09574091011071906, International Journal of Logistics Management, 21(2), 161-179 (2010).

Eyaa, S., Ntayi, J. y Namagebe, S., Collaborative relationships and SME supply chain performance, doi: 10.1108/20425961201000018, World Journal of Entrepreneurship, Management Sustainable Development, 6(3), 233-245 (2010).

Fantazy, K. y Salem, M., The value of strategy and flexibility in new product development: The impact on performance, doi: 10.1108/JEIM-10-2014-0102, Journal of Enterprise Information Management, 29(4), 525-548 (2016).

Firdauz, A.M., Sapri, M. y Mohammad, I., Facility management knowledge development in Malaysia Added value in hospitality managerial competency, doi: 10.1108/F-04-2013-0034, Facilities, 33(1/2), 99-118 (2015).

Fletcher, L. y Polychronakis, Y., Capturing knowledge management in the supply chain, doi: 10.1108/14502190710826059, EuroMed Journal of Business, 2(2), 191-207 (2007).

Galvao, C., Wang, G. y Mileski, J., Public-private interests and conflicts in ports: A content analysis approach, doi: 10.1016/j.ajsl.2016.03.002, Asian Journal of Shipping and Logistics, 32(1), 13-22 (2016).

Hair, J., Anderson, R. y otros 2 autores, Análisis Multivariante, Prentice Hall, Madrid, España (1999).

Hamid, N., Use balanced scorecard for measuring competitive advantage of infrastructure assets of state-owned ports in Indonesia: Case in Pelindo IV, Indonesia, doi: 10.1108/JMD-12-2016-0313, Journal of Management Development, 37(2), 114-126 (2018).

Kaliani, V., Chandran, V. y Muhamm, A., Supply chain practices and performance: The indirect effects of Supply Chain Integration, doi: 10.1108/BIJ-03-2015-0023, International Journal of Process Management and Benchmarking, 23(6), 1445-1471 (2016).

Kearney, A., Harrington, D. y Kelliher, F., Executive capability for innovation: the Irish seaports sector, doi: 10.1108/EJTD10-2017-0081, European Journal of Training and Development, 42(5/6), 342-361 (2018).

Kwak, D., Seo, Y. y Mason, R., Investigating the relationship between supply chain innovation, risk management capabilities and competitive advantage in global supply chains, doi:10.1108/IJOPM-06-2015-0390, International Journal of Operations y Production Management, 38(1), 2-21 (2018).

Kwok, W. y Kian, S., Technology use and learning characteristics of students in higher education: Do generational differences exist?, doi: 10.1111/bjet.12161, British Journal of Educational Technology, 46(6), 725-738 (2015).

Lambe, P., The unacknowledged parentage of knowledge management, doi: /10.1108/13673271111119646, Journal of Knowledge Management, 15(2), 175-197 (2011).

Laperche, B. y Liu, Z., SMEs and knowledge-capital formation in innovation networks: a review of literature, doi: 10.1186/2192-5372-2-21, Journal of Innovation and Entrepreneurship, 2(21), 1-16 (2013).

Lee, P., Kwon, O. y Ruan, X., Sustainability challenges in maritime transport and logistics industry and its way ahead, doi: 10.3390/su11051331, Sustainability (Switzerland), 11(5) (2019).

Liao, Y., y Barnes, J., Knowledge acquisition and product innovation flexibility in SMEs, doi: 10.1108/BPMJ-05-2014-0039, Business Process Management Journal, 21(6), 1257-1278 (2015).

Li, S., Rao, S. y otros 2 autores, Development and validation of a measurement instrument for studying supply chain management practices, doi: 10.1016/j. jom2005.01.002, Journal of Operations Management, 618-641 (2005).

López, C. y Meroño, A., Strategic knowledge management, innovation and performance, doi:10.1016/j.ijinfomgt.2011.02.003, International Journal of Information Management, 31, 502-509 (2011).

Martínez, I., Munduate, L. y Medina F.J. Efectividad de los Patrones de Influencia, Psicothema, 20 (3), 369+ (2008).

Martínez, L., Ferreira, A. y Can, A., Consultant-client relationship and knowledge transfer in small- and medium-sized enterprises change processes, doi: 10.1177/0033294116639429, Psychological Reports, 118(2), 608-625 (2016).

Massingham, P., An evaluation of knowledge management tools: Part 1 - managing knowledge resources, doi: 10.1108/JKM-11-2013-0449, Journal of Knowledge Management, 18(6), 1075-1100. (2014).

Oliva, F. L., Gomes, M. H. y otros 2 autores, The integration between knowledge management and dynamic capabilities in agile organizations, doi: 10.1108/MD-06-2018-0670, Management Decision 57(8) 1969-1979 (2019). 
Rodríguez, M., y Ruiz, M., Atenuación de la asimetría y de la curtosis de las puntuaciones observadas mediante transformaciones de variables: Incidencias sobre la estructura factorial. Psicológica: Revista de Metodología y Psicología Experimental, 29(2), 205-227 (2008).

Simangunsong, E., Hendry, L. y Steveson, M., Managing supply chain uncertainty with emerging ethical issues, doi: 10.1108/IJOPM-12-2014-0599, International Journal of Operations and Production Management, 36(10), 1-33 (2016).

Sobel, M. E., Some new results on indirect effects and their standard errors in covariance structure models, doi: 10.2307/270922, Sociological methodology, 16, 159-186 (1986).

Vasconcelos, A., Dilemmas in knowledge management, doi: 10.1108/01435120810869165, Library Management, 29(4/5), 422-443 (2008).

Vieera, E., Corporate risk in family businesses under economic crisis, doi: 10.15446/innovar.v24n53.43909, Innovar, 24(53), 61-73 (2014).

Wynarczyk, P., Open innovation in SMEs. A dynamic approach to modern entrepreneurship in the twenty-first century, doi: 10.1108/14626001311326725, Journal of Small Business and Enterprise Development, 20(2), 258-278 (2013).

Yousif L.A. y Hassan S., Knowledge management strategies, innovation and organisational performance: An empirical study of the Iraqi MTS, doi: 10.1108/09727981311327767, Journal of Advances in Management Research, 10(1), 58-71 (2013).

Zortea, E., Darroch, J. y Matear, S., Business orientations and innovation in small and medium sized enterprises, doi: 10.1007/s11365-011-0170-7, International Entrepreneurship and Management Journal, 8(2), 145-164 (2012). 